\title{
LEVANTAMENTO DAS INTERNAÇÕES E ÓBITOS POR CAUSAS AMBIENTAIS NO MUNICÍPIO DE PALMAS/TOCANTINS DE 2008 A 2015
}

$\begin{array}{llllll}\text { SURVEY OF HOSPITALIZATIONS } & \text { AND } & \text { DEATHS } & \text { BY } \\ \text { ENVIRONMENTAL CAUSES IN THE } & \text { MUNICIPALITY } & \text { OF } \\ \text { PALMAS/TOCANTINS FROM 2008 TO } 2015 & & & \end{array}$

\author{
Andressa Paula Silva Coelho ${ }^{1}$, Dulce Mourthé Starling Pinheiro ${ }^{1}$, Evandro \\ Leite Bitencourt ${ }^{1}$, Paulo Martins Reis Júnior².
}

\begin{abstract}
RESUMO
Objetivo: Realizar um levantamento das internações e óbitos por causas ambientais no município de Palmas/Tocantins- TO de 2008 a 2015. Métodos: Análise dos dados obtidos através da plataforma online do Departamento de Informática do Sistema Único de Saúde (DATASUS). Foram selecionadas de acordo com a 10a edição da Classificação Estatística Internacional de Doenças e Problemas Relacionados com a Saúde (CID-10), os subgrupos: W65 a W74, Afogamento e submersão acidental; códigos de X20 a X29, Contato com animais e plantas venenosas; e códigos de X30 a X39, Exposição às forças da natureza. Resultados: Foram registrados: 69 óbitos de 2008 a 2015 por causas ambientais, dentre os quais $75,36 \%$ por afogamento e submersão acidentais; $20,29 \%$ por contato com animais e plantas venenosos e $4,35 \%$ por exposição às forças da natureza. $82,60 \%$ dos óbitos pertenciam ao sexo masculino $17,40 \%$ ao feminino. A faixa etária mais acometida por afogamentos e submersão foi de20 a 29 anos e por contato com animais e plantas venenosas foi de40 a 59 anos. Em relação à escolaridade, em $57,97 \%$ não há informação. 0 local mais comum dos óbitos foram os hospitais. E em $86 \%$ das internações a causa foi contato com animais e plantas venenosos. Conclusões: Observou-se que os óbitos por causas ambientais têm perfil similar ao das vítimas por causas externas no Brasil. Assim, medidas preventivas educacionais e de prevenção de saúde podem reduzir o número de internações e óbitos. Constatou-se uma limitação referente à coleta de dados, demonstrando a fragilidade do sistema de informações de saúde e necessidade de melhorias no preenchimento dessas informações. Nesse sentido, há implicações diretas na interpretação dos resultados e consequentes implementações de medidas preventivasassim como há a necessidade de mais artigos e estudos na área.
\end{abstract}

Palavras-chave: Internação hospitalar; Óbito; Sistemas de informação.

\begin{abstract}
Citação: Coelho APS, Pinheiro DMS, Bitencourt EL, Reis Júnior PM (2017) Levantamento das internações e óbitos por causas ambientais no município de Palmas/Tocantins de 2008 a 2015. Revista de Patologia do Tocantins, 4(4): 7-12.
\end{abstract}

Instituição: ${ }^{1}$ Acadêmico(a) de Medicina, Universidade Federal do Tocantins, Tocantins, Brasil; ${ }^{2}$ Docente, Médico Cirurgião, Universidade Federal do Tocantins, Tocantins, Brasil.

\section{Autor correspondente: Andressa Paula Silva Coelho; andressainstitucional@gmail.com}

Editor: Guedes V. R. Medicina, Universidade Federal do Tocantins, Brasil.

Publicado: 01 de dezembro de 2017.

Direitos Autorais: (c) 2017 Coelho et al. Este é um artigo de acesso aberto que permite o uso, a distribuição e a reprodução sem restrições em qualquer meio, desde que o autor original e a fonte sejam creditados.

Conflito de interesses: os autores declararam que não existem conflitos de interesses.

\begin{abstract}
Objective: Carry out a survey of hospitalizations and deaths by environmental resources in the municipality of Palmas/Tocantins-TO from 2008 to 2015. Methods: Data analysis obtained through the online platform of the Unified Health System's Information Technology Department (DATASUS). The following subgroups were selected according to the 10th International Statistical Classification of Diseases and Related Health Problems (ICD-10): W65 to W74, Accidental drowning and submersion; codes from X20 to X29, Contact with poisonous animals and plants; and codes from X30 to X39, Exposure to the forces of nature. Results: 69 deaths were recorded from 2008 to 2015 due to environmental causes, among which $75.36 \%$ were accidental drowning and submersion; $20.29 \%$ by contact with poisonous animals and plants and $4.35 \%$ by exposure to the forces of nature. $82.60 \%$ of deaths with men and $17.40 \%$ with women. The most common age group in drowning and submersion was 20 to 29 years and in contact with poisonous animals and plants 40 to 59 years old. Regard to schooling, in $57.97 \%$ there is no information. The most common place of death were hospitals. And in $86 \%$ of hospitalizations the cause for contact with poisonous animals and plants. Conclusions: It was observed that the deaths due to environmental resources have a profile likethat of the victims due to external causes in Brazil. Thus, educational priventive and health prevention measures can reduce the number of hospitalizations and deaths. There was a limitation regarding data collection, demonstrating a fragility of the health information system and the need for improvements in the information. In this sense, there are direct implications in the interpretation of results and consequent implementations of preventive measures as there is a need for more articles and studies in the area.
\end{abstract}

Keywords: Hospitalization; Death; Information systems. 


\section{INTRODUÇÃO}

Os acidentes ocasionados por fatores ambientais, com risco ou não de óbito, englobam afogamentos, queimaduras, lesões por deslizamentos, ictismo, dentre outros eventos como as descargas elétricas. Os acidentes por causas ambientais compreendem um subgrupo que pode ser incluído no grupo de acidentes por causas externas. ${ }^{1}$. As causas externas, desde 1980, mostraram-se em segundo lugar no índice de morte no Brasil ${ }^{2}$.

Os afogamentos são de extrema importância ao se analisar os óbitos por causas ambientais. Esse tipo de óbito passa por três fases, sendo elas: a fase de defesa, a fase de resistência e a fase de exaustão. A fase de defesa possui dois períodos: o de surpresa e o de dispneia. A fase de resistência é representada pela parada dos movimentos respiratórios como um mecanismo de defesa. A fase de exaustão ocorre quando a resistência pela exaustão termina e começa uma profunda inspiração, iniciando-se o processo de asfixia com perda de consciência, insensibilidade em alguns casos, convulsões e por fim a morte ${ }^{3}$.

O afogamento é a terceira causa de mortes involuntárias relacionadas a lesões em todo o mundo, de acordo com a Organização Mundial da Saúde, representando cerca de 370 mil mortes anuais e $7 \%$ de todas as mortes relacionadas a lesões. Dentro deste contexto, os mais afetados são os países de baixa e média renda, representando cerca de $91 \%$ das mortes involuntárias porafogamento ${ }^{4}$.

Outro evento relacionado aos óbitos por causas ambientais são as queimaduras, que consistem em lesões de pele ou de outro tecido orgânico primariamente causado por um trauma térmico ou outro trauma agudo. Uma queimadura ocorre quando algumas ou todas as células da pele ou de outros tecidos são destruídas por líquidos quentes (escaldaduras), sólidos quentes (queimaduras por contato) ou por chamas (queimaduras por chamas). Lesões de pele ou de outros tecidos orgânicos devido à radiação, radioatividade, eletricidade, fricção ou contato com substâncias químicas também são identificadas como queimaduras ${ }^{5}$.

Os deslizamentos/escorregamentos são fenômenos que também podem ser incluídos nas causas de óbitos por causas externas associadas às forças das naturezas, segundo o capítulo XX da 10ạ edição da Classificação Estatística Internacional de Doenças e Problemas Relacionados com a Saúde $(C I D-10)^{6}$. As mudanças climáticas globais podem acarretar os deslizamentos e consequentes desastres por soterramentos. Além disso, outros fatores estão associados como a geomorfologia e ação humana na ocupação de determinado solo. Esses deslizamentos ocasionam prejuízos de caráter não apenas ambiental, mas também social e econômico ${ }^{7}$. O número de óbitos por deslizamentos no Brasil entre 1988 a 2008 foi de $1.861^{8}$.

Segundo o documento referencial da Secretaria Especial de Comunicação Social ${ }^{9}$, o número de acidentes relacionados a animais venenosos é cada vez maior, especialmente os associados às arraias devido às condições hídricas do estado do Tocantins (TO). Embora a letalidade seja menor comparada aos outros acidentes, há uma preocupação em virtude das hospitalizações e dos possíveis prejuízos funcionais às vítimas ${ }^{9}$.
As arraias são peixes encontrados no fundo dos ambientes marítimos ou fluviais, apresentam formato e tamanho variáveis. Possuem hábito carnívoro (alimentando-se de diferentes seres vivos como outros peixes e crustáceos). São marcadas pela presença de ferrões dorsais revestidos por substâncias tóxicas ${ }^{10}$. Essas arraias podem provocar acidentes graves, relatados, principalmente, em pescadores e banhistas que acidentalmente pisam-nas, uma vez que ficam soterradas nas areias. Ocorrem, portanto, ferimentos perfuro-cortantes em geral na região do tornozelo e pés; e as toxinas liberadas ocasionam intenso processo doloroso ${ }^{11}$.

Outros acidentes traumáticos podem ocorrer por meio de ferimentos também perfuro-cortantes provocados pelos peixes denominados de piranhas, que vivem em água doce. Essas lesões são laceradas e ocasionam sangramentos. Cerca de $55 \%$ dos acidentes em pescadores de água doce foram causados por peixes traumatogênicos, como as piranhas; segundo estudo envolvendo acidentes em rios e lagos do Brasil ${ }^{12}$.

Tendo em vista a importância dos acidentes por causas ambientais associados aos óbitos por causas externas no território brasileiro como um todo, é essencial compreender o problema no município de Palmas a fim de identificar soluções. Nesse sentido, o estudo em questão tem como objetivo realizar um levantamento das internações e óbitos por causas ambientais no município de Palmas/TO de 2008 a 2015, o que permite um suporte para ações preventivas e resolutivas voltadas para a temática.

\section{MÉTODOS}

Este artigo baseia-se em dados de domínio público disponibilizados pelo Ministério da Saúde do Brasil, através de plataforma online do Departamento de Informática do Sistema Único de Saúde (DATASUS). Para o levantamento e análise de dados na plataforma, foram acessados os Sistemas de Informações Hospitalares e sobre Mortalidade (SIM/SIH).

As modalidades de acidentes por causas ambientais foram selecionadas de acordo com o CID-10 ${ }^{6}$. Foram considerados os seguintes grupos do CID-10: códigos de W65 a W74, Afogamento e submersão acidental; códigos de X20 a $X 29$, Contato com animais e plantas venenosas; e códigos de X30 a X39, Exposição às forças da natureza.

Cada grupo de CID-10 citado anteriormente engloba vários subgrupos. Esses subgrupos, bem como os critérios de inclusão e exclusão de cada grande grupo do CID-10 estão descritos abaixo:

Grande grupo: W65-W74: Afogamento e submersão acidental

Exclui: afogamento e submersão devidos a: acidente (s) de transporte (V01-V99), por água (V90, V92) e cataclisma (X34-X39).

W65- Afogamento e submersão durante banho em banheira

W66- Afogamento e submersão consecutiva a queda dentro de uma banheira

W67- Afogamento e submersão em piscina 
W68- Afogamento e submersão consequente a queda dentro de uma piscina

W69.- Afogamento e submersão em águas naturais

Inclui: córrego; curso d'água; lago; mar aberto e rio.

W70-Afogamento e submersão consequentes a queda dentro de águas naturais

W73- Outros afogamentos e submersão especificados

Inclui: reservatório, tanque de resfriamento.

W74- Afogamento e submersão não especificados

Inclui: afogamento sem outra especificação (SOE), queda dentro da água SOE.

\section{Grande grupo: X20-X29: Contato com animais e plantas venenosas}

Inclui: liberação de veneno por meio de dentes inoculadores, pelos espinhos, tentáculos e outros órgãos de inoculação de veneno, mordeduras e picadas venenosas substâncias químicas liberadas por: animais e insetos.

Exclui: ingestão de animais ou de plantas venenosas (X49)

X20- Contato com serpentes e lagartos venenosos

Inclui: caiçara (jararaca), cascavel, cobra, monstro de Gila, serpente: marinha e víbora.

Exclui: cobra não-venenosa (W59) e lagarto (nãovenenoso) (W59).

X21- Contato com aranhas venenosas

Inclui: tarântula e viúva negra.

X22- Contato com escorpiões

X23- Contato com abelhas, vespas e vespões

Inclui: vespa americana.

X24- Contato com centopeias e miriápodes venenosas (tropicais)

X25- Contato com outros artrópodes venenosos

Inclui: formiga, lagarta.

X26- Contato com animais e plantas marinhos venenosos

Inclui: água-viva, anêmona do mar, coral, espinho de ouriço do mar, nematocistos, outros animais ou plantas marinhas, pepino do mar.

Exclui: animais marinhos não-venenosos (W56), serpentes marinhas (X20).

$\mathrm{X} 27$ - Contato com outros animais venenosos especificados

X28- Contato com outras plantas venenosas especificadas

Inclui: inoculação de venenos ou de toxinas através da pele por espinhos ou outras estruturas vegetais.

Exclui: ferimento puncionante SOE causado por picada de espinhos de plantas (W60), ingestão de plantas venenosas (X49).

X29-Contato com animais ou plantas venenosas, sem especificação.

Inclui: mordedura venenosa SOE e picada (venenosa) SOE.
Grande grupo: X30-X39: Exposição às forças da natureza

X30- Exposição a calor natural excessivo

Inclui: calor excessivo como causa de insolação, exposição ao calor SOE.

Exclui: calor excessivo de fonte artificial (W92).

X31- Exposição a frio natural excessivo

Inclui: exposição (a) (ao): condições climáticas, frio SOE

frio excessivo como causa de pé ou mão de imersão e/ou úlceras de frio SOE.

Exclui: contato com ou inalação de: gás líquido (W93), neve carbônica (gelo seco) (W93) e frio de origem artificial (W93).

X32- Exposição à luz solar

X33- Vítima de raio.

Exclui: fogo causado por raio (X00-X09), traumatismo por queda de árvore ou outro objeto, causado por raio (W20).

X34- Vítima de terremoto

X35- Vítima de erupção vulcânica

X36- Vítima de avalanche, desabamento de terra e outros movimentos da superfície terrestre

Inclui: deslizamento da lama de natureza cataclísmica.

Exclui: acidente de transporte em que ocorre a colisão com avalanche ou desabamento de terra não-emmovimento (V01-V99) e terremoto (X34).

X37- Vítima de tempestade cataclísmica

Inclui: aguaceiro, chuva torrencial, ciclone, enchente, furacão, inundação, macaréu causado por tempestade, nevasca, tempestade de neve, tornado, veículo de transporte carregado para fora da estrada pela tempestade.

Exclui: acidente de transporte que ocorre após a tempestade (V01-V99) e ruptura de barragem ou de outra obra causando movimento da superfície terrestre (X36).

X38- Vítima de inundação

Inclui: inundação: cataclísmica, resultante do degelo da neve, provocada por uma tempestade a distância, resultante diretamente de uma tempestade.

Exclui: macaréu [ondas de maré]: SOE (X39), causado por tempestade (X37), ruptura de barragem ou de outra obra causando movimento da superfície terrestre (X36).

X39- Exposição a outras forças da natureza e às não especificadas

Após o recolhimento dos dados pelo instrumento de coleta, as frequências absolutas e relativas anuais de óbitos e internações, por cada grande grupo do CID-10 especificados, foram agrupados em planilhas do software Excel ${ }^{\circledR}$ para Windows 10.

Analisaram-se também as variáveis como sexo, faixa etária, escolaridade e local de ocorrência.

Além disso, o presente artigo tem como critérios de inclusão todos os dados que estavam disponíveis para a coleta 
dentro do período pré-estabelecido de 6 meses. Os critérios de exclusão são os citados acima de acordo com o CID-10.

\section{RESULTADOS}

Foram registrados 69 óbitos de 2008 a 2015 por causas ambientais de acordo com os grupos do CID-10, no município de Palmas. Desses, 75,36\% foram por afogamento e submersão acidentais; $20,29 \%$ por contato com animais e plantas venenosos e 4,35\% por exposição às forças da natureza. Os anos de 2010 e 2013 apresentaram respectivamente 13 e 11 óbitos por causas ambientais, em valores absolutos. Em contraste no ano de 2011, ocorreram 2 óbitos no município de Palmas por causas ambientais (Tabela 1 e Figura 1).

Figura 1. Óbitos por Causas Ambientais em Palmas/TO, 2008- 2015. Óbitos por causas ambientais de 2008 a 2015 na cidade Palmas/TO

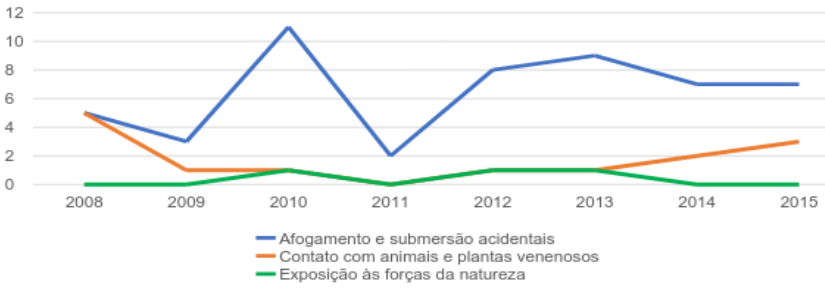

Fonte: DATASUS. Informações de Saúde, Sistema de Informações sobre Mortalidade (SIM).

Tabela 1. Distribuição de Óbitos por Causas Ambientais em Palmas/TO, 2008- 2015.

\begin{tabular}{|c|c|c|}
\hline \multirow{2}{*}{ Variáveis } & \multicolumn{2}{|c|}{ Óbitos (n=69) } \\
\hline & $\mathrm{n}$ & $\%$ \\
\hline \multicolumn{3}{|l|}{ Grupo CID-10 } \\
\hline W65-W74 Afogamento e submersão acidentais & 52 & $75,36 \%$ \\
\hline $\begin{array}{l}\text { Contato com animais e } \times 20-\times 29 \text { Contato animais } \\
\text { e plantas venenosos }\end{array}$ & 14 & $20,29 \%$ \\
\hline X30-X39 Exposição às forças da natureza & 3 & $4,35 \%$ \\
\hline \multicolumn{3}{|l|}{ Sexo } \\
\hline Masculino & 57 & $82,60 \%$ \\
\hline Feminino & 12 & $17,40 \%$ \\
\hline \multicolumn{3}{|l|}{ Faixa etária (anos) } \\
\hline Menor de um ano & 2 & $2,89 \%$ \\
\hline 1 a 4 & 9 & $13,04 \%$ \\
\hline 5 a 9 & 8 & $11,59 \%$ \\
\hline 10 a 14 & 4 & $5,79 \%$ \\
\hline 15 a 19 & 6 & $8,74 \%$ \\
\hline 20 a 29 & 11 & $15,94 \%$ \\
\hline 30 a 39 & 8 & $11,59 \%$ \\
\hline 40 a 49 & 11 & $15,94 \%$ \\
\hline 50 a 59 & 4 & $5,79 \%$ \\
\hline 60 a 69 & 3 & $4,35 \%$ \\
\hline 70 a 79 & 2 & $2,89 \%$ \\
\hline 80 anos ou mais & 1 & $1,45 \%$ \\
\hline \multicolumn{3}{|l|}{ Escolaridade (em anos de estudo) } \\
\hline Nenhuma & 3 & $4,35 \%$ \\
\hline 1 a 3 & 10 & $14,49 \%$ \\
\hline 4 a 7 & 10 & $14,49 \%$ \\
\hline 8 a 11 & 5 & $7,25 \%$ \\
\hline 12 anos ou mais & 1 & $1,45 \%$ \\
\hline Ignorado & 40 & $57,97 \%$ \\
\hline \multicolumn{3}{|l|}{ Local de ocorrência } \\
\hline Hospital & 27 & $39,13 \%$ \\
\hline Outro estabelecimento de saúde & 1 & $1,45 \%$ \\
\hline Domicílio & 1 & $1,45 \%$ \\
\hline Via pública & 1 & $1,45 \%$ \\
\hline Outros & 39 & $56,52 \%$ \\
\hline
\end{tabular}

Fonte: DATASUS. Informações de Saúde, Sistema de Informações sobre Mortalidade (SIM).

O sexo masculino apresentou número óbitos de $82,60 \%$ do total em relação ao sexo feminino $(17,40 \%)$ pelas mesmas causas ambientais, no período 2008-2015, sendo que essa diferença foi ainda mais expressiva nogrupo afogamento e submersão acidentais (Figura 2).

Figura 2. Sexo dos óbitos por Causas Ambientais em Palmas/TO, 2008- 2015.

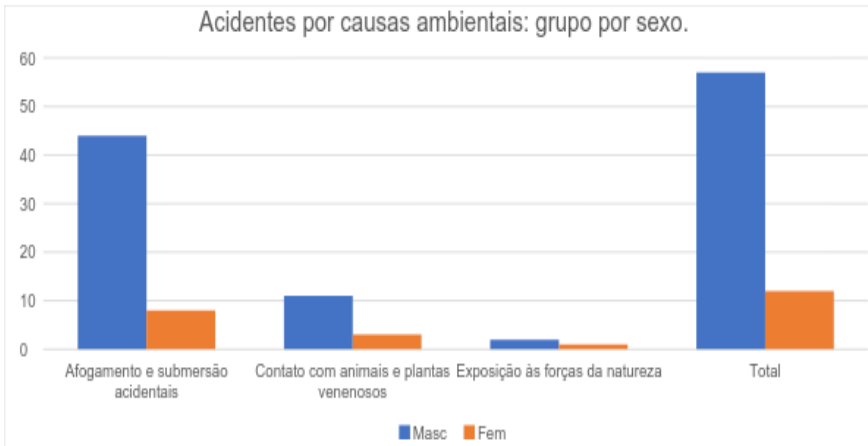

Fonte: DATASUS. Informações de Saúde, Sistema de Informações sobre Mortalidade (SIM).

Quanto à faixa etária, foram as crianças e adolescentes (1 a 19 anos), bem como os adultos jovens (20 a 29 anos) e adultos até 49 anos, os mais acometidos no total (82,60\%). Por grupo do CID-10, os adultos jovens foram os mais acometidos por afogamento e submersão acidentais (17,30\%); as faixas etárias de 40-49 anos e 50-59 anos empataram em maior número de óbitos por contato com animais e plantas venenosos (cada um representa $21,43 \%$ do total do grupo); e em exposição às forças da natureza não houve uma faixa etária com um número prevalente (Figura 3).

Figura 3. Faixa etária dos óbitos por Causas Ambientais em Palmas/TO, 2008- 2015.

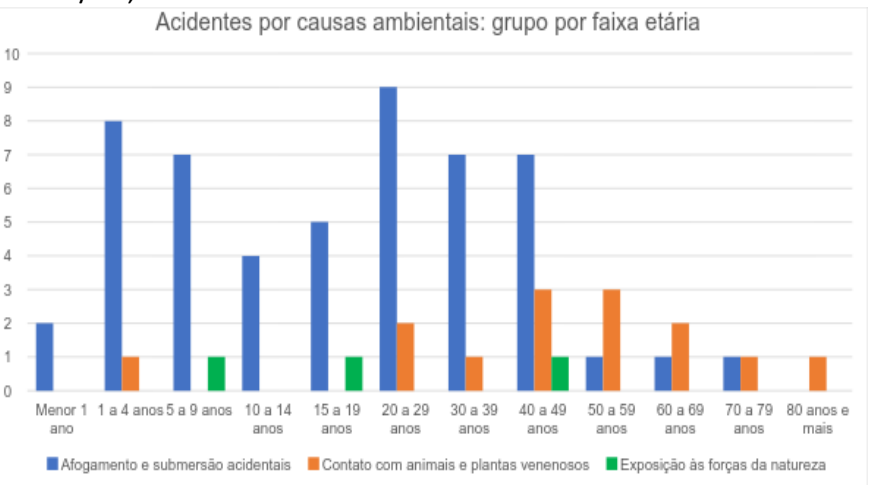

Fonte: DATASUS. Informações de Saúde, Sistema de Informações sobre Mortalidade (SIM).

Quanto à escolaridade, 4,35\% das vítimas apresentavam nenhum nível de escolaridade; $14,49 \%$ apresentavam 1 a 3 anos de estudos; 14,49\% apresentavam 4 a 7 anos de estudo; 1,45\% apresentavam 12 anos ou mais; e em $57,97 \%$ dos casos não havia essa informação, dado ignorado (Tabela 1 e Figura 4).

Figura 4. Escolaridade dos óbitos por Causas Ambientais em Palmas/TO, 2008- 2015. 
Acidentes por causas ambientais: grupo por escolaridade

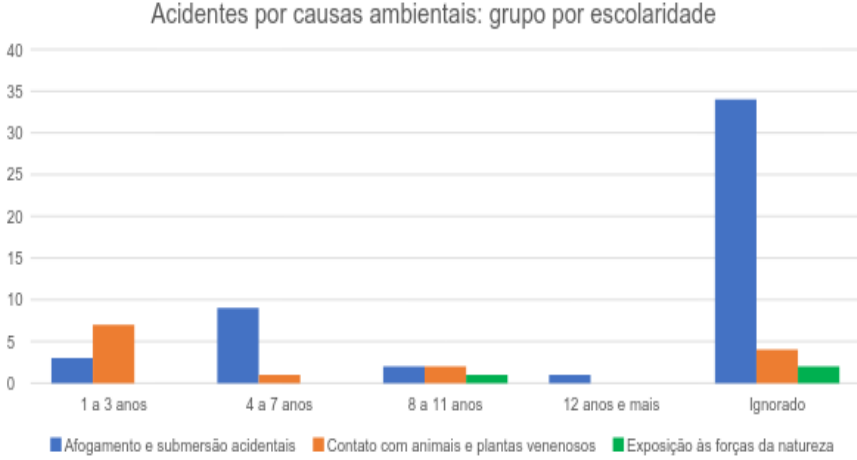

Fonte: DATASUS. Informações de Saúde, Sistema de Informações sobre Mortalidade (SIM).

Ao analisar os óbitos por causas ambientais de acordo com o local de ocorrência, os dados mais expressivos representam os óbitos em hospitais $(39,13 \%)$ e outros $(56,52 \%)$ lugares não especificados (Figura 5).

Figura 5. Local de ocorrência dos óbitos por Causas Ambientais em Palmas/TO, 2008- 2015.

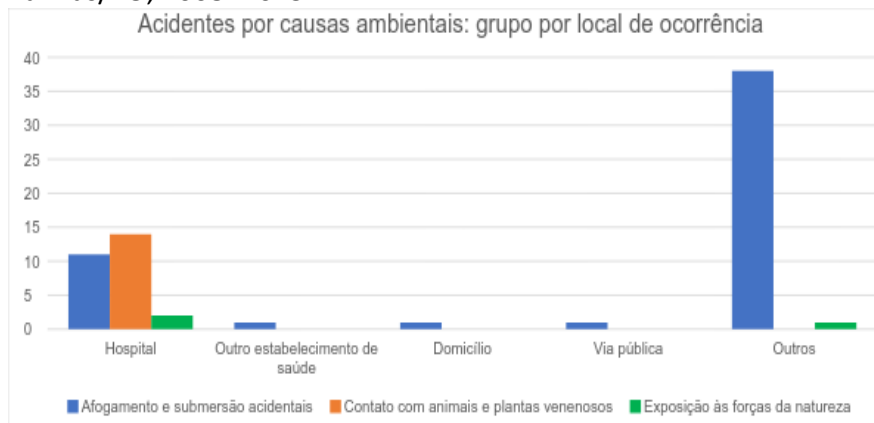

Fonte: DATASUS. Informações de Saúde, Sistema de Informações sobre Mortalidade (SIM).

Observa-se do gráfico (Figura 6) que ocorreu um total de 50 internações por causas ambientais no município de Palmas, no período de 2008 a 2015. Dessas internações, 86,0\% foram por contato com animais e plantas venenosos; $14,0 \%$ por afogamento e submersão acidentais. Não foi registrada nenhuma internação por exposição às forças da natureza. Das internações por contato com animais e plantas venenosos, no ano de 2015 foi encontrado maior número de internações, 14 em valor absoluto.

Figura 6. Internações por Causas Ambientais em Palmas/TO, 20082015.

Internações por causas ambientais de 2008 a 2015 na cidade de Palmas/TO

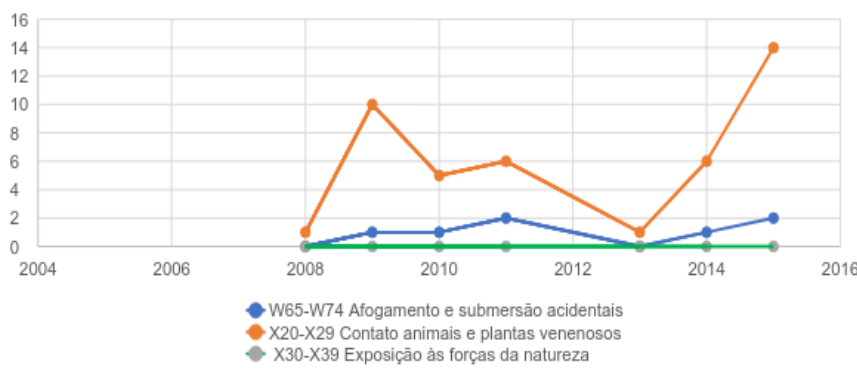

Fonte: DATASUS. Informações de Saúde, Sistema de Informações Hospitalares (SIH).

\section{DISCUSSÃO}

De acordo com os dados apresentados, $75,36 \%$ dos óbitos foram por afogamento e submersão acidentais o que demonstra a expressividade desse tipo de óbito por causas ambientais e corrobora com os dados de outros estudos epidemiológicos ${ }^{4}$.

Em relação ao número de vítimas por causas ambientais por sexo, observou-se que o sexo masculino foimais acometido, com $82,60 \%$ do total de óbitos no período. Isso significa que ocorreram cerca de 5 vítimas do sexo masculino para cada vítima do sexo feminino. Esses resultados acompanham a proporção de óbitos por afogamento $e$ submersão acidentais do estado do Tocantins, o que permite associação uma vez que há uma forte expressividade desse fator causal dentro das causas ambientais ${ }^{13}$. A proporcionalidade de 5:1 reafirma um resultado nacional dos óbitos por causas externas em que essa proporção é ainda maior, 8 vítimas do sexo masculino para cada vítima do sexo feminino; o que pode ser atribuído aos modelos culturais, em que o homem é mais exposto a situações de risco ${ }^{14}$.

Quanto à idade dos indivíduos que morreram por causas ambientais, as faixas de 1 a 9 anos; 20 a 29 e adultos até 49 anos são as mais acometidas (82,60\%). Essa informação demonstra uma tendência dos óbitos por causas externas de seremmais prevalentes nas crianças, adolescentes e adultos jovens, pois esses grupos estão diretamente associados a fatores de risco como a vulnerabilidade, impulsividade e uso de drogas lícitas ou não. Além disso, é relevante o impacto negativo socioeconômico desses óbitos não apenas para os centros de saúde e mercado de trabalho, mas também aos familiares das vítimas ${ }^{15}$.

Em relação ao nível de escolaridade, observou-se que em 33,33\% dos casos o nível de escolaridade era inferior a 7 anos de estudo. Esse dado revela a forte associação entre baixo nível de escolaridade e a incidência de óbitos, mostrando a necessidade de intervenções na área de educação. Percebeu-se também um valor elevado de dados ignorados (57,97\%)em relação à escolaridade, o que demonstra a fragilidade dos sistemas de informações e interfere diretamente na interpretação do resultados ${ }^{13}$. Consequentemente, há um prejuízo sob a perspectiva do presente estudo e na instituição de intervenções práticas a partir do mesmo.

Quanto aos óbitos por local de ocorrência, observouse uma expressiva quantidade de óbitos por causas ambientais em hospitais e em locais não especificados, enquanto em domicílio, via pública ou outro estabelecimento de saúde não foram registrados óbitos por contato com plantas e animais venenosos, nem por exposição às forças da natureza, apenas por afogamentos e submersão acidentais e em menor quantidade. Tal fato pode ser tanto consequência de uma necessidade de a população buscar primeiro a atenção terciária em saúde bem como pode ser atribuído a uma subnotificação de mortes que nem chegam a ser registradas justamente pelos locais em que ocorreram, podendo não ter quem encontrasse ou presenciasse o acidente. Contudo, apesar de se poder pensar na subnotificação como uma das explicações, ainda não há um estudo específico que corrobore tal argumento. Existem apenas alguns estudosque revelam subnotificação em regiões específicas do Brasil por causas também particulares ${ }^{16,17}$. 
Os acidentes com animais e plantas venenosos nem sempre são associados à letalidade, no entanto representam uma preocupação em virtude de suas consequências como as hospitalizações e o prejuízo funcional das vítimas, que por sua vez correlacionam-se com o impacto econômico, principalmente ${ }^{9}$. Nesse sentido, essa preocupação é reforçada pelo fato de86,0\% das internações por causas ambientais serem atreladas a acidentes com animais e plantas venenosos.

\section{CONCLUSÕES}

Com este estudo, estabeleceram-se o perfil dos óbitos por acidentes de causas ambientais, e as causas de acidentes envolvendo fatores ambientais que levaram ao maior número de internações no município de Palmas, no período de 2008 a 2015. Concluiu-se que esse perfil dos óbitos é similar ao das vítimas por causas externas no Brasil, tendo em vista que esse último grupo engloba as causas ambientais. Nesse sentido ações de promoção da saúde poderiam reduzir os fatores de riscos dos grupos mais expostos a esses acidentes, diminuindo, portanto o número de internações e óbitos.

Constatou-se ainda uma limitação referente à coleta de dados, em virtude da elevada taxa de dados ignorados pelo sistema de informações de saúde, demonstrando sua fragilidade e necessidade de melhorias no preenchimento dessas informações.

\section{REFERÊNCIAS BIBLIOGRÁFICAS}

1. Gonsaga RAT, Rimoli CF, Pires EA, Zogheib FS, Fujino MVT, Cunha MB. Avaliação da mortalidade por causas externas. Rev Col Bras Cir. Rio de Janeiro, 2012; 39(4): 263-267.

2. Santos AMR, Moura MEB, Nunes BMVT, Leal CFS, Teles JBM. Perfil das vítimas de trauma por acidente de moto atendidas em um serviço público de emergência. Cad saúde pública. Rio de Janeiro, 2008;24(8):1927-1938.

3. França GV. Medicina Legal.10.ed. Rio de Janeiro: Guanabara Koogan, 2015.

4. Tyler MD, Richards DB, Reske-Nielsen C, Saghafi O, Morse EA, Carey $\mathrm{R}$, Jacquet GA. The epidemiology of drowning in low- and middleincome countries: a systematic review. BMC Public Health. London, 2017; 17: 413-420.

5. Peck MD. Epidemiology of burns throughout the world. Part I: Distribution and risk factors. Rewiew Burns. 2011; 37(7): 1087-1100.

6. Organização Mundial de Saúde. CID-10 Classificação Estatística Internacional de Doenças e Problemas Relacionados à Saúde. São Paulo: Edusp; 2007.

7. Tominaga LK, Santoro J, Amaral R. Desastres naturais: conhecer para prevenir. São Paulo: Instituto Geológico; 2009.

8. Instituto de Pesquisas Tecnológicas. Banco de dados de mortes por escorregamentos no Brasil. Banco de Dados Digital. São Paulo: IPT; 2009.

9. Secretaria de Comunicação Social- SECOM. Risco de acidentes com animais peçonhentos cresce no período de praias, Sesau registrou mais de dois mil casos. Disponível em: <http://secom.to.gov.br/noticia/220518/>. Acesso em: 21 de outubro de 2017.

10. Carvalho MR, Lovejoy NR, Rosa RS. Family Potamotrygonidae (river stingrays). In: Reis RE et al. Check list of the freshwater fishes of South and Central America. In: Morfometria de raias continentais (Chondrichthyes, Potamotrygonidae) do alto rio Paraná, Brasil. Acta Sci Biol Sci.Maringá (PR), 2007; 4: 413-419.
11. Haddad Jr V, Garrone ND, Paula JBN, Marques FPL, Barbaro KC. Freshwater stingrays: study of epidemiologic, clinic and therapeutic aspects based on 84 envenomings in humans and some enzymatic activities of the venom. In: Morfometria de raias continentais (Chondrichthyes, Potamotrygonidae) do alto rio Paraná, Brasil. Acta Sci Biol Sci. Maringá (PR), 2007; 4: 413-419.

12. Haddad Jr V. Avaliação Epidemiológica, Clínica e Terapêutica de Acidentes Provocados por Animais Peçonhentos Marinhos na região Sudeste do Brasil. In: HADDAD Jr., V. Animais aquáticos de importância médica no Brasil.Rev Soc Bras Med Trop. Minas Gerais: 2003; 36(5): 591-597.

13. Rodrigues AL, Carvalho RS, Cuellar PMG. Perfil epidemiológico dos óbitos por afogamento no estado do Tocantins no período de 2010 a 2014. Rev de Patologia do Tocantins. Tocantins, 2017; 4(3):3-8.

14. Moura EC, Gomes R, Falcão MTC, Schwarz E, Neves ACM, Santos $W$. Desigualdades de gênero na mortalidade por causas externas no Brasil, 2010. Ciência \& Saúde Coletiva. Rio de Janeiro, 2015; 20(3): 779-788.

15. Matos K, Martins CBG. Mortalidade por causas externas em crianças, adolescentes e jovens: uma revisão bibliográfica. Rev Espaço para a Saúde. Londrina (PR), 2013; 14(2): 82-93.

16. Binder MCP, Cordeiro R. Sub-registro de acidentes do trabalho em localidade do Estado de São Paulo, 1997. Revde Saúde Pública. São Paulo, 2003; 37(4): 409-416.

17. Correa PRL, Assunção AA. A subnotificação de mortes por acidentes de trabalho: estudo de três bancos de dados. Epidemiol Serv Saúde. Brasília, 2003; 12(4): 203-212. 